\title{
A note on admissibility when the credit line is infinite
}

\author{
Sara Biagini * $\quad$ Mihai Sîrbu ${ }^{\S}$
}

November 29, 2010

\begin{abstract}
In the context of expected utility maximization for utilities defined on the whole real line, we define a new class of admissible strategies in terms of dynamic bounds on losses under the historical measure $\mathbb{P}$. More precisely, the loss control is given by a $\mathbb{P}$-martingale which is compatible with the preferences of the investor. The main result is the Ansel-Stricker-type Lemma 3.3 which shows that the admissible strategies are supermartingales under all sigma-martingale measures $\mathbb{Q}$ with finite relative entropy, therefore allowing for a duality theory for the optimization problem.
\end{abstract}

Key words: utility maximization - non locally bounded semimartingale - investment opportunity supermartingale property - duality theory

JEL Classification: G11, G12

Mathematics Subject Classification (2000): primary 60G48, 60G44, 49N15, 91B28; secondary 46E30, 46N30, 91B16.

\section{Introduction}

We consider the "classic" frictionless model for the evolution of $d+1$ traded (liquid) assets. We assume that the risk-free asset (the money market account) is constant, or equivalently, that the interest rates are zero. This is a customary assumption in the utility maximization literature which does not restrict generality as long as interest rates are deterministic. The $d$ risky assets, denoted by $S=\left(S^{1}, \ldots, S^{d}\right)$, are assumed to be a (vector-valued, càdlàg) semimartingale on the stochastic basis $\left(\Omega, \mathcal{F},\left(\mathcal{F}_{t}\right)_{0 \leq t \leq T}, \mathbb{P}\right)$ which verifies the usual assumptions of completeness and right-continuity.

The trading period is the interval $[0, T]$. A continuous-time trading (or investment) strategy is a predictable, $S$-integrable and $\mathbb{R}^{d}$-valued process $H$, where $H_{t}=\left(H_{t}^{1}, \ldots, H_{t}^{d}\right)$ represents the number of shares of each risky asset held by the agent in the infinitesimal interval $[t, t+d t]$. If the strategy is (self)-financed by borrowing/investing in the risk-free money market, given the initial endowment $x$ the (càdlàg) wealth process $X$ of the investor evolves according to

$$
X_{t}=x+\int_{0}^{t} H_{s} d S_{s}, \quad 0 \leq t \leq T .
$$

We denote by $\mathcal{I}(x)$ the set of all such stochastic integrals with initial value $x$. Usually, some restrictions must be imposed in order for trading strategies to have financial meaning. The main goal of the present note is to define the precise restrictions needed to pass from the full set $\mathcal{I}(x)$ to a smaller set of so called admissible strategies $\mathcal{X}(x)$ in the context of optimal investment. We model the preferences of an investor with time horizon $T$ by expected utility from terminal wealth. A utility function is a function $U:(a, \infty) \rightarrow \mathbb{R}$ for some $-\infty \leq a<\infty$, which is nondecreasing and concave. While further additional assumptions are usually made on the utility function $U$, they are not needed for our purposes. The optimal investment problem can be formulated as

$$
u(x):=\sup _{X \in \mathcal{X}(x)} \mathbb{E}\left[U\left(X_{T}\right)\right]
$$

\footnotetext{
*University of Pisa. Email: sara.biagini@ec.unipi.it

$\S$ University of Texas at Austin. Email: sirbu@math.utexas.edu. The research of this author was supported in part by the National Science Foundation under Grants DMS 0802681 and DMS 0908441.
} 
where $u$ denotes the value function, also known as indirect utility. In what follows, $X^{*}(x)$ or simply $X^{*}$ indicates the optimal wealth process (if it exists) and $H^{*}$ the optimal trading strategy, $X^{*}=x+\int H^{*} d S$.

As it is well known, doubling strategies or similar arbitrage schemes should be excluded from the class of investment strategies. Therefore, the very definition of the domain $\mathcal{X}(x)$ is a delicate issue. The nature of the utility function $U$ itself leads to two different cases:

If $-\infty<a<\infty$, the agent tolerates only to lose a fixed amount of money (no more than $x-a$ ). Typical examples are the logarithmic or power utility. The natural admissibility condition is to require wealth processes to be uniformly bounded below by $a$, which means that the agent never exceeds his/her finite credit line (see e.g. [KS99], [BF08] and the discussions and bibliography cited there). Under additional hypotheses on the utility function $U$ and a no arbitrage assumption, in this case the optimization problem (1) can be solved by duality methods and the optimal strategy is admissible.

The case $a=-\infty$ corresponds to an infinite credit line. The archetypal example is the exponential utility, $U(x)=-\frac{1}{\gamma} e^{-\frac{1}{\gamma} x}$. Here the definition of admissibility is a much more delicate issue, and this is exactly where the contribution of the present note lies. In Section 3, admissibility is defined in terms of a loss control which is a martingale under the historical measure $\mathbb{P}$ and satisfies a natural compatibility condition with the preferences of the investor. To the best of our knowledge, this is the first attempt to use a $\mathbb{P}$-martingale as a bound on losses of admissible strategies.

The paper is organized as follows: Section 2 reviews existing definitions of admissibility for $a=-\infty$, pointing out the technical difficulties related to this case. This review plays a double role: it motivates the introduction of our new admissible class $\mathcal{X}^{a d}(x)$ and allows us to compare it with the previously defined admissible classes. Section 3 contains the definition of $\mathcal{X}^{a d}(x)$ and its properties: the main result is Lemma 3.3 stating the so-called "supermartingale property". In Section 4 we present an important example that can be solved completely using the new definition of admissibility. Section 5 concludes with a thorough comparison of the newly defined class with the literature. For the sake of completeness, some needed Lemmas are presented in the Appendix.

\section{Existing definitions of admissible strategies when $a=-\infty$}

The literature is roughly split into two main branches: economically reasonable definitions of strategies, stated in terms of bounds on the losses, versus the good mathematical definition, given through the so-called "supermartingale property" of the wealth processes. Our goal is to define a class of strategies/integrals which is a good compromise between these two directions, analyzed hereafter.

\subsection{Admissibility defined by bounds on losses}

The first direction focuses on the economic interpretation of admissibility of the strategies $H$, by introducing a pathwise loss control on the wealth processes $X=x+\int H d S$. In case $S$ is locally bounded, Schachermayer [Sch01] defines as admissible the wealth processes (and relative strategies) which are uniformly bounded from below by some constant, in analogy to the definition when $a$ is finite:

$$
\mathcal{X}^{b b}(x)=\left\{X \in \mathcal{I}(x) \mid(\exists) c>0 \quad X_{t} \geq-c, \quad 0 \leq t \leq T\right\} .
$$

This amounts to having a finite credit line which is dependent on the strategy. Using this definition of admissibility, a duality formula is proven in [Sch01]. The drawback is that the supremum in (1) is usually not attained over the admissible domain, or $X^{*} \notin \mathcal{X}^{b b}(x)$. The analysis in [Sch01] actually works for the more general case of $S$ being sigma-bounded, namely when there exist a scalar positive integrand $\varphi$ such that $\int \varphi d S$ is (well defined and) bounded. The concept of sigma localization was introduced in [Kal02], and sigma-boundedness was used in the context of utility maximization in [KS06].

When $S$ is non locally bounded, the set $\mathcal{X}^{b b}(x)$ may be too small to set up the optimization problem (1), even if one does not necessarily care about the optimizer being admissible. The following simple example in [Sch01] shows that $\mathcal{X}^{b b}(x)$ may not contain any non-trivial strategy.

Example 2.1. Consider a one period market model, with $\mathcal{F}_{0}$ trivial and let $S_{0}=0, S_{1}$ be Gaussian. Then, any integral is of the form $x+h S_{1}, h \in \mathbb{R}$, and is thus bounded from below only if $h=0$. 
The example above can be easily modified so that the stock is positive but unbounded above. In this case, long positions are admissible in the sense of $\mathcal{X}^{b b}(x)$, but short positions are not. It is still unreasonable to preclude a-priori any short position from the optimization problem (1), especially if the probabilities of large losses are very small.

Therefore, for unbounded stock prices, one must necessarily relax the admissibility condition, as first addressed in Biagini and Frittelli [BF05, BF08]. The solution proposed there is the following. Consider a control random variable $W$ which is compatible with the preferences, that is it verifies the $U$-integrability condition

$$
\mathbb{E}[U(-\alpha W)]>-\infty
$$

either for all constants $\alpha>0$ or for (the weaker requirement of) some $\alpha>0$. Denote by $\mathcal{W}$ the set of random variables $W$ which satisfy (2). For a fixed $W \in \mathcal{W}$, define the class of admissible wealth processes

$$
\mathcal{X}^{W}(x)=\left\{X \in \mathcal{I}(x) \mid(\exists) c>0 \quad X_{t} \geq-c W \text { for all } t \in[0, T]\right\}
$$

When $W=1$ then $\mathcal{X}^{W}(x)=\mathcal{X}^{b b}(x)$. As may be easily derived from the mathematical details provided in the Appendix, a combination of Ansel-Stricker Lemma [AS94] and Fenchel inequality shows that $\mathcal{X}^{W}(x) \subset \mathcal{X}^{\sup }(x)$, where the set of strategies $\mathcal{X}^{\text {sup }}(x)$ is defined by:

Definition 2.2. The class $\mathcal{X}^{\text {sup }}(x)$ is the set of wealth processes $X \in \mathcal{I}(x)$ which are supermartingales under any probability measure $\mathbb{Q}$ which is a sigma-martingale measure for $S$

$$
\mathbb{Q} \in \mathcal{M}_{\sigma}=\left\{\mathbb{Q}^{\prime} \ll \mathbb{P} \mid S \text { is a sigma-martingale under } \mathbb{Q}^{\prime}\right\}
$$

and has finite $V$-entropy

$$
\mathbb{Q} \in \mathcal{P}_{V}=\left\{\mathbb{Q} \ll \mathbb{P} \mid(\exists) y>0, \quad \mathbb{E}\left[V\left(y \frac{d \mathbb{Q}}{d \mathbb{P}}\right)\right]<\infty\right\}
$$

Here $V(y)=\sup _{x}\{U(x)-x y\}$ is the convex conjugate of $U$.

We remind the reader that a process $S$ is a sigma-martingale if there exist a scalar positive integrand $\varphi$ such that $\int \varphi d S$ is (well defined and) a martingale. This is another instance of sigma localization as defined in [Kal02].

The set $\mathcal{M}_{\sigma} \cap \mathcal{P}_{V}$ is actually the dual domain of the optimization problem (1). If, for the same fixed $W \in \mathcal{W}$, some restrictions in terms of $W$ are imposed on the jumps of $S$, (there exists a non trivial set of $W$-controlled strategies), then the dual domain $\mathcal{M}_{\sigma} \cap \mathcal{P}_{V}$ satisfies a closure property, allowing for a complete duality theory.

In spite of the higher flexibility, compared to wealth bounded below by a constant, there is still no guarantee about the admissibility of the optimal process $X^{*}$ - the examples in [BF05] (as well as the example in Section 5) show that

$$
X^{*} \notin \mathcal{X}^{W}(x)
$$

may happen.

Remark 2.3. Even when $S$ is locally (or sigma)-bounded, an alternative to $\mathcal{X}^{b b}(x)$ is

$$
\mathcal{X}^{U}(x)=\left\{X \in \mathcal{I}(x) \mid(\exists) \alpha>0, \quad \mathbb{E}\left[U\left(-\alpha \sup _{0 \leq t \leq T}\left(X_{t}^{-}\right)\right)\right]>-\infty\right\}=\cup_{W \in \mathcal{W}} \mathcal{X}^{W}(x) .
$$

Duality still works, since $\mathcal{M}_{\sigma} \cap \mathcal{P}_{V}$ is closed. In addition, $\mathcal{X}^{U}(x)$ has a much better chance of capturing the optimal strategy than $\mathcal{X}^{b b}(x)$ (see Section 4). This means that the enlargement of the admissible domain that was originally needed in order to accommodate some simple trading strategies if stock-prices are unbounded, may actually be mathematically useful even for the case of bounded $S$, by making the optimization problem (1) well-posed. An evident drawback of the class $\mathcal{X}^{U}(x)$ is that the control is not dynamic, as the maximal loss is known only at the terminal time $T$. 


\subsection{Admissibility defined through the supermartingale property}

The second direction focuses more on giving a mathematically good definition, in the sense that the optimal process will surely be admissible. The set $\mathcal{X}^{\text {sup }}(x)$ in Definition 2.2 is therefore considered as the set of admissible strategies. For locally (or sigma) bounded $S$, the "supermartingale strategies" are used in the Six Authors' paper [6AU] and Kabanov and Stricker [KS02] for exponential $U$; in Schachermayer [Sch03] for general $U$ and in the more recent Owen and Zitkovic [OZ07] for the case when there is random endowment. We recall that when $S$ is locally bounded, $\mathcal{M}_{\sigma}$ are simply the local martingale measures for $S$, so that $\mathcal{M}_{\sigma} \cap \mathcal{P}_{V}$ are the local martingale measures with finite entropy. The supermartingale strategies can be used also when $S$ is not locally bounded, as shown by Biagini and Frittelli [BF07].

The main point in favor of the supermartingale strategies is that the optimal process $X^{*}$ belongs to this class,

$$
X^{*} \in \mathcal{X}^{\text {sup }}(x),
$$

but there is a price to pay. Such a definition is not easy to interpret economically, as it is given in terms of the dual set of probability measures $\mathcal{M}_{\sigma} \cap \mathcal{P}_{V}$. Moreover, realistic market models are incomplete and thus the description of the whole $\mathcal{M}_{\sigma} \cap \mathcal{P}_{V}$ is often impossible. Consequently, checking admissibility with respect to this definition is practically unfeasible.

\section{The new class of admissible strategies $\mathcal{X}^{a d}(x)$}

As already mentioned, our goal is to define a new class of strategies/integrals which reconciles as much as possible these two different directions of the current literature. We are thus interested in a class of admissible strategies which:

1. has a clear financial interpretation, therefore is of the type in Subsection 2.1 (control on losses)

2. the control on losses is adapted (dynamic)

3. is larger than any economically reasonable class defined so far (for example in [Sch01], [BF05], [BF08]) to capture as much as possible the optimizer. In other words, we want a class of admissible strategies larger than the class $\mathcal{X}^{U}(x)$ defined in (4), even in the locally bounded case.

It turns out that the good notion of admissibility is a loss control which is a martingale under the historical measure $\mathbb{P}$, plus a compatibility condition with the preferences in the spirit of Biagini and Frittelli.

Definition 3.1. The set of admissible processes $\mathcal{X}^{a d}(x)$ is the set of wealth processes $X \in \mathcal{I}(x)$ such that there exists a (positive) martingale $W$ under the historical measure $\mathbb{P}$ such that

$$
X_{t} \geq-W_{t}, \quad 0 \leq t \leq T,
$$

and for some $\alpha>0$

$$
\mathbb{E}\left[U\left(-\alpha W_{T}\right)\right]>-\infty .
$$

The martingale property of the dynamic loss control $W=\left(W_{t}\right)_{0 \leq t \leq T}$ means that bounds on losses at earlier times are just conditional expectations under historical measure $\mathbb{P}$ of the bound on terminal loss. Condition (5) is the compatibility with respect to the preferences mentioned above. A first property is:

Lemma 3.2. The set $\mathcal{X}^{a d}(x)$ is convex.

Proof. Consider $X^{i} \in \mathcal{X}^{a d}(x), i=1 \ldots n$. For each $i$, denote by $W^{i}$ the positive martingale which controls the losses of $X^{i}$ and denote by $\alpha_{i}$ a compatibility coefficient satisfying (5). Fix nonnegative weights $\lambda_{i}, \sum_{i} \lambda_{i}=1$. Then, $Y:=\sum_{i} \lambda_{i} X^{i}$ satisfies

$$
Y \geq-W, \quad W:=\sum_{i} \lambda_{i} W^{i}
$$


and $W$ is a martingale, which satisfies (5) with $\alpha=\min _{i} \alpha_{i}$ :

$$
E\left[U\left(-\alpha W_{T}\right)\right] \geq \sum_{i} \lambda_{i} E\left[U\left(-\alpha W_{T}^{i}\right)\right] \geq \sum_{i} \lambda_{i} E\left[U\left(-\alpha_{i} W_{T}^{i}\right)\right]>-\infty,
$$

where the first inequality is Jensen's inequality and the second follows from the monotonicity of $U$.

This definition is dynamic, as the control on losses is a process, and admissibility can be checked directly under $\mathbb{P}$. In addition, $\mathcal{X}^{a d}(x)$ is stable under stopping (an easy consequence of Jensen's inequality) and

$$
\mathcal{X}^{U}(x) \subset \mathcal{X}^{a d}(x) .
$$

The inclusion above follows directly from Definition (4). In fact, by setting $W_{T}:=\sup _{0 \leq t \leq T}\left(X_{t}^{-}\right)$and $W_{t}=E_{t}\left[W_{T}\right]$, one obtains a $\mathbb{P}$-martingale which controls the losses of $X$ :

$$
X_{t} \geq-\sup _{0 \leq s \leq t}\left(X_{s}^{-}\right)=-E_{t}\left[\sup _{0 \leq s \leq t}\left(X_{s}^{-}\right)\right] \geq-E_{t}\left[W_{T}\right] .
$$

Therefore, $\mathcal{X}^{a d}(x)$ includes any class described in Section 2.1. The result below states that $\mathcal{X}^{\text {ad }}(x)$ also enjoys the "super-martingale property":

Lemma 3.3. Any $X \in \mathcal{X}^{a d}(x)$ is a supermartingale under all $\mathbb{Q} \in \mathcal{M}_{\sigma} \cap \mathcal{P}_{V}$.

Proof. Fix $\mathbb{Q} \in \mathcal{M}_{\sigma} \cap \mathcal{P}_{V}$ and denote by $Z$ its density process. Let $y>0$ such that $\mathbb{E}\left[V\left(y Z_{T}\right)\right]<\infty$. An application of the Fenchel inequality at time $t$ gives

$$
U\left(-\alpha W_{t}\right)+\alpha y W_{t} Z_{t} \leq V\left(y Z_{t}\right),
$$

so that

$$
W_{t} Z_{t} \leq \frac{V\left(y Z_{t}\right)-U\left(-\alpha W_{t}\right)}{\alpha y} .
$$

Denoting by $M_{t}=\frac{V\left(y Z_{t}\right)-U\left(-\alpha W_{t}\right)}{\alpha y}$, Jensen's inequality implies that $M:=\left(M_{t}\right)_{0 \leq t \leq T}$ is a nonnegative submartingale under $\mathbb{P}$ that controls $W Z$ :

$$
0 \leq W Z \leq M .
$$

The family of nonnegative random variables $\left(M_{\tau}\right)_{\tau}$ is uniformly integrable, since $M_{\tau} \leq E_{\tau}\left[M_{T}\right]$ and the family $\left(E_{\tau}\left[M_{T}\right]\right)_{\tau}$ is uniformly integrable. For each stopping time $\tau \leq T$,

$$
\mathbb{E}_{\mathbb{Q}}\left[W_{\tau} 1_{\left\{W_{\tau}>c\right\}}\right]=\mathbb{E}\left[Z_{\tau} W_{\tau} 1_{\left\{W_{\tau}>c\right\}}\right] \leq \mathbb{E}\left[M_{\tau} 1_{\left\{W_{\tau}>c\right\}}\right] .
$$

Now, $\mathbb{P}\left(W_{\tau}>c\right) \leq \mathbb{E}\left[W_{\tau}\right] / c=\mathbb{E}\left[W_{T}\right] / c$, so

$$
\lim _{c \rightarrow \infty} \sup _{\tau} \mathbb{E}_{\mathbb{Q}}\left[W_{\tau} 1_{\left\{W_{\tau}>c\right\}}\right]=0 .
$$

In other words, $\left(W_{\tau}\right)_{\tau}$ is a uniformly integrable family under $\mathbb{Q}$. Since $X$ is bounded below by $W$, $\left(X_{\tau}^{-}\right)_{\tau \leq T}$ is also a uniformly integrable family under $\mathbb{Q}$. Lemma 6.1 in the Appendix implies that $X$ is a local martingale and a supermartingale under $\mathbb{Q}$.

Remark 3.4. In case $\mathbb{Q} \sim \mathbb{P}$, the proof above can be made even shorter, as $\frac{M}{Z}$ is well defined, and a $\mathbb{Q}$-submartingale.

The supermartingale property is very desirable because of its economic budget implications.

Corollary 3.5. The class $\mathcal{X}^{a d}(x)$ verifies a budget constraint with respect to all sigma-martingale measures compatible with the preferences, i.e. for all $\mathbb{Q} \in \mathcal{M}_{\sigma} \cap \mathcal{P}_{V}$ and all $\tau \leq T$ stopping times,

$$
\mathbb{E}_{\mathbb{Q}}\left[X_{\tau}\right] \leq x .
$$

The budget constraint (6), which is a direct consequence of our definition of admissibility, allows for a duality approach to the optimization problem (1). We present below an example which can be completely solved using the new Definition. 


\section{An example}

We consider a variation of the classical Merton problem with exponential utility and a Lévy asset. More precisely, consider the utility function $U(x)=-\frac{1}{\gamma} e^{-\gamma x}, x \in \mathbb{R}$, for some $\gamma>0$ and the asset $\left(L_{t}\right)_{0 \leq t \leq T}$ being a Lévy process with characteristic triplet $(\mu, \sigma, \nu(d y))$ with respect to the cut-off function $h(y)=\overline{\mathbb{I}}_{\{|y| \leq 1\}}$. The filtration is the natural enlarged filtration generated by $L$.

Consider now the optimal investment problem

$$
\sup _{X \in \mathcal{X}^{\operatorname{ad}}(x)} \mathbb{E}\left[U\left(X_{T}\right)\right]
$$

where $\mathcal{X}^{a d}(x)$ was defined in Definition 3.1 and $L$ plays the role of $S$. At a formal level, the solution to the problem is well known. More precisely it is well known that the optimal investment strategy is a constant $H^{*} \equiv \theta^{*}$ where $\theta^{*}$ is chosen to maximize the expected utility over all possible $\theta$. The wealth process corresponding to a fixed constant strategy $\theta$ is $X^{\theta}=x+\theta L$, and the expected utility is therefore given by the well-known moment generating function of Lévy processes

$$
\mathbb{E}\left[U\left(X_{T}^{\theta}\right)\right]=-\exp (-\gamma x+g(\theta) T),
$$

where the Lévy exponent $g(\theta)$ is formally defined by

$$
g(\theta) \triangleq-\mu \gamma \theta+\frac{1}{2} \sigma^{2} \gamma^{2} \theta^{2}+\int_{-\infty}^{\infty}\left(e^{-\gamma \theta y}-1+\gamma \theta y \mathbb{I}_{\{|y| \leq 1\}}\right) \nu(d y) .
$$

The function $g$ is a convex and proper function, i.e. $g>-\infty$. In addition, the proper domain is actually equal to the set $D \subset \mathbb{R}$ of those $\theta$ for which the measure $\mathbb{I}_{\{|y|>1\}} \nu(d y)$ has a finite exponential moment, modulo the scaling $-\gamma$, which means

$$
D \triangleq\left\{\theta \mid \int_{|y|>1} e^{-\gamma \theta y} \nu(d y)<\infty\right\} .
$$

Note the proper domain $D$ is nonempty since $0 \in D$. Using the convexity of the exponential function, we know that $e^{-\gamma \theta y}-1+\gamma \theta y \geq 0$, so

$$
g(\theta) \geq-\mu \gamma \theta+\frac{1}{2} \sigma^{2} \gamma^{2} \theta^{2}+\int_{|y|>1}\left(e^{-\gamma \theta y}-1\right) \nu(d y)
$$

Since the exponential is positive and $\nu(\{|y|>1\})<\infty$, the convex function $g$ is coercive, which reads $g(\theta) \rightarrow \infty$ as $\theta \rightarrow \infty$. We can now use Fatou Lemma in the representation (8) for $|y|>1$ and the Dominated Convergence Theorem for $|y| \leq 1$ to also obtain that the function $g$ is lower semicontinuous. This implies that there exists a $\theta^{*} \in D$ where $g$ attains its global minimum. Actually $\theta^{*}$ is unique by strict convexity.

The whole solution of the problem is an exercise involving the moment generating functions of Lévy processes, and is based on the following well known lemma:

Lemma 4.1. Let $R$ be a random variable, and assume that there exist $\alpha_{1}<\alpha_{2}$ such that

$$
f(\alpha) \triangleq \mathbb{E}\left[e^{\alpha R}\right]<\infty \text { for } \alpha \in\left(\alpha_{1}, \alpha_{2}\right) .
$$

Then $\mathbb{E}\left[|R| e^{\alpha R}\right]<\infty$ for $\alpha \in\left(\alpha_{1}, \alpha_{2}\right)$. In addition, $f$ is continuously differentiable on $\left(\alpha_{1}, \alpha_{2}\right)$ and

$$
f^{\prime}(\alpha)=\mathbb{E}\left[R e^{\alpha R}\right] \text { for } \alpha \in\left(\alpha_{1}, \alpha_{2}\right) .
$$

We now assume that the global minimum of $g$ is attained in the interior of the proper domain (which is implicitly assumed non-empty).

Assumption 4.2. Assume that $\theta^{*} \in \operatorname{int}(D) \neq \emptyset$. 
Under Assumption 4.2, using Lemma 4.1 for the measure $\mathbb{I}_{\{|y|>1\}} \nu(d y)$ rather than the random variable $R$, together with Dominated Convergence Theorem for $|y| \leq 1$ we obtain that the function $g$ defined in (8) is finite and continuously differentiable in $\operatorname{int}(D)$ and

$$
g^{\prime}(\theta)=-\mu \gamma+\sigma^{2} \gamma^{2} \theta+\int_{-\infty}^{\infty}\left(-\gamma y e^{-\gamma \theta y}+\gamma y \mathbb{I}_{\{|y| \leq 1\}}\right) \nu(d y) \text { for } \theta \in \operatorname{int}(D),
$$

where all the integral above is well defined and finite. This means that Assumption 4.2 in particular implies that $g^{\prime}\left(\theta^{*}\right)=0$.

Proposition 4.3. Fix $x \in(-\infty, \infty)$. Under the Assumption 4.2 the process $X^{*}=X^{\theta^{*}}$ attains the maximum in the optimization problem (7). More precisely, we have

1. $X^{*} \in \mathcal{X}^{a d}(x)$

2. $\mathbb{E}\left[U\left(X_{T}^{*}\right)\right] \geq \mathbb{E}\left[U\left(X_{T}\right)\right]$ for any $X \in \mathcal{X}^{a d}(x)$.

Proof. 1. Up to the initial capital $x$ the process $X^{*}$ is a Lévy process. More precisely, $X=x+L^{*}$, where $L^{*} \triangleq \theta^{*} L$ is a Lévy process with jump measure

$$
\nu^{*}(A)=\nu\left(\frac{A}{\theta^{*}}\right), \quad A \subset \mathbb{R}-\{0\} .
$$

Since $\theta^{*} \in D$, then we have

$$
\int_{|y|>1} e^{-\gamma y} \nu^{*}(d y)<\infty .
$$

Let us consider the Lévy process $N$, obtained from $L^{*}$ by removing the positive jumps of size strictly larger than one. The characteristic triplet of $N$ is $\left(\mu, \sigma, \nu_{N}(d y)\right)$, with $\nu_{N}(d y)=\mathbb{I}_{\{y<1\}} \nu^{*}(d y)$.

Therefore (9) means that the measure $\mathbb{I}_{\{|y|>1\}} \nu_{N}(d y)$ has finite absolute $\gamma$-exponential moment, which, thanks to Theorems 25.3 in [Sato], is equivalent to $\mathbb{E}\left[e^{\gamma\left|N_{t}\right|}\right]<\infty$ for each $t$. Theorem 25.18 in [Sato] ensures that

$$
\mathbb{E}\left[e^{\gamma \sup _{0 \leq t \leq T}\left|N_{t}\right|}\right]<\infty .
$$

Taking into account that $N \leq L^{*}$, so $\left(L^{*}\right)^{-} \leq|N|$, we easily obtain

$$
\mathbb{E}\left[e^{\gamma \sup _{0 \leq t \leq T}\left(L_{t}^{*}\right)^{-}}\right]<\infty .
$$

As an immediate consequence, we get (using the set $\mathcal{X}^{U}(x)$ defined in Remark 2.3) that

$$
X^{*} \in \mathcal{X}^{U}(x) \subset \mathcal{X}^{a d}(x),
$$

despite the fact that $X^{*} \notin \mathcal{X}^{b b}(x) \subset \mathcal{X}^{U}(x)$.

2. Second, since $U^{\prime}(x)=e^{-\gamma x}$, we see that $U^{\prime}\left(X_{T}^{*}\right)=e^{-\gamma x-\gamma \theta^{*} L_{T}}$. Since $\theta^{*} \in D$, then

$$
\mathbb{E}\left[U^{\prime}\left(X_{T}^{*}\right)\right]<\infty,
$$

so

$$
U^{\prime}\left(X_{T}^{*}\right)=c \frac{d \mathbb{Q}}{d \mathbb{P}}
$$

where $\mathbb{Q}$ is a probability measure equivalent to $\mathbb{P}$. Lemma 4.1 implies that

$$
\mathbb{E}\left[U^{\prime}\left(X_{T}^{*}\right)\left|X_{T}^{*}\right|\right]<\infty .
$$

If $V$ is the convex conjugate of the utility function $U$, then we have

$$
V\left(c \frac{d \mathbb{Q}}{d \mathbb{P}}\right)=U\left(X_{T}^{*}\right)-c X_{T}^{*} \frac{d \mathbb{Q}}{d \mathbb{P}} \in L^{1}
$$


which means that the measure $\mathbb{Q}$ has finite entropy. The density process of the measure $\mathbb{Q}$ is actually given by

$$
Z_{t}=\frac{e^{\theta^{*} L_{t}}}{e^{t g\left(\theta^{*}\right)}} .
$$

For any $0 \leq s \leq t \leq T$, we have, from Assumption 4.2 and Lemma 4.1 that

$$
\mathbb{E}\left[e^{\theta^{*}\left(L_{t}-L_{s}\right)}\left(L_{t}-L_{s}\right)\right]=g^{\prime}\left(\theta^{*}\right)(t-s) e^{g\left(\theta^{*}\right)(t-s)}=0 .
$$

Using the above relation we obtain

$$
\mathbb{E}\left[Z_{t}\left(L_{t}-L_{s}\right) \mid \mathcal{F}_{s}\right]=\frac{e^{\theta^{*} L_{s}}}{e^{t g\left(\theta^{*}\right)}} \mathbb{E}\left[e^{\theta^{*}\left(L_{t}-L_{s}\right)}\left(L_{t}-L_{s}\right) \mid \mathcal{F}_{s}\right]=\frac{e^{\theta^{*} L_{s}}}{e^{t g\left(\theta^{*}\right)}} \mathbb{E}\left[e^{\theta^{*}\left(L_{t}-L_{s}\right)}\left(L_{t}-L_{s}\right)\right]=0 .
$$

This means that $\mathbb{Q}$ is actually a martingale measure (not just local) for $L$, so $\mathbb{Q} \in \mathcal{M}_{\sigma} \cap \mathcal{P}_{V}$. Now, for any $X \in \mathcal{X}^{a d}(x)$, we have

$$
U\left(X_{T}\right)-U\left(X_{T}^{*}\right) \leq U^{\prime}\left(X_{T}^{*}\right)\left(X_{T}-X_{T}^{*}\right)
$$

Integrating, we obtain

$$
\mathbb{E}\left[U\left(X_{T}\right)-U\left(X_{T}^{*}\right)\right] \leq c \mathbb{E}_{\mathbb{Q}}\left[X_{T}-X_{T}^{*}\right] .
$$

According to Corollary 3.5, we have $\mathbb{E}_{\mathbb{Q}}\left[X_{T}\right] \leq x$ (this is exactly where our main result is used). At the same time, the process $X^{*}=x+\theta^{*} L$ is a true martingale with respect to the measure $\mathbb{Q}$, so $\mathbb{E}_{\mathbb{Q}}\left[X_{T}^{*}\right]=x$. We can therefore conclude that

$$
\mathbb{E}\left[U\left(X_{T}\right)\right]-\mathbb{E}\left[U\left(X_{T}^{*}\right)\right]=\mathbb{E}\left[U\left(X_{T}\right)-U\left(X_{T}^{*}\right)\right] \leq 0, \text { for all } X \in \mathcal{X}^{a d}(x),
$$

and the proof is complete.

\section{The counter-example of Schachermayer and conclusions}

For the sake of completeness, we would like to recall the reader of a counter-example in [Sch03] (Proposition 2, Section 3). In order to emphasize the dependence of the admissible domain (be it either $\mathcal{X}^{\text {sup }}$ or $\mathcal{X}^{a d}$ ) on both the traded assets $S$ and the initial capital $x$, we will use the notation $\mathcal{X}(S, x)$.

Example 5.1. Consider the exponential utility $U(x)=-e^{-x}$. There exists a financial market with two locally bounded risky assets $S=\left(S^{1}, S^{2}\right)$ satisfying $S_{0}^{1}=S_{0}^{2}=1$ and such that the optimal strategy in the investment problem

$$
\sup _{X \in \mathcal{X}^{b b}(S, 1)} \mathbb{E}\left[U\left(X_{T}\right)\right]=\max _{X \in \mathcal{X}^{s u p}(S, 1)} \mathbb{E}\left[U\left(X_{T}\right)\right],
$$

is to buy and hold the first asset, completely disregarding the second. In addition, the optimal strategy is a supermartingale under any martingale measure, so we have

$$
X^{*}=S^{1} \in \mathcal{X}^{\text {sup }}(S, 1) .
$$

However, if the second asset is withdrawn from the market, and one considers the optimal investment problem in the first asset alone,

$$
\sup _{X \in \mathcal{X}^{b b}\left(S^{1}, 1\right)} \mathbb{E}\left[U\left(X_{T}\right)\right]=\sup _{X \in \mathcal{X}^{\sup }\left(S^{1}, 1\right)} \mathbb{E}\left[U\left(X_{T}\right)\right]
$$

then

$$
S^{1} \notin \mathcal{X}^{\text {sup }}\left(S^{1}, 1\right)
$$

and the new optimal strategy is to invest everything in the money market account. 
Remark 5.2. In the example above, $X^{*}=S^{1} \notin \mathcal{X}^{a d}(S, 1)$. Indeed, if the strategy $X^{*}=S^{1}$ were in the admissible set $\mathcal{X}^{a d}(S, 1)$, it would also be admissible for the market in which only $S^{1}$ is traded, so we would obtain

$$
X^{*}=S^{1} \in \mathcal{X}^{a d}\left(S^{1}, 1\right) \subset \mathcal{X}^{\text {sup }}\left(S^{1}, 1\right),
$$

according to our main result, Lemma 3.3. Since the above inclusion does not hold, we therefore have that $\mathcal{X}^{a d}(S, 1) \subsetneq \mathcal{X}^{\text {sup }}(S, 1)$.

To conclude the paper, we have constructed a new class of admissible strategies which:

a- has a clear financial interpretation, with restrictions imposed in terms of dynamic pathwise loss controls

b- is larger than previous economically meaningful classes, namely $\mathcal{X}^{U}(x) \subset \mathcal{X}^{a d}(x)$

c- in some important examples (like Section 4) the optimizer is actually admissible

d- is (still) smaller than the whole class of "supermartingale strategies", so it may not always contain the optimal strategy (see Example 5.1 and Remark 5.2).

The message of item d- should not be regarded as a negative result due to our specific choice of admissibility. In a general semimartingale model it is actually impossible to completely reconcile an economically meaningful definition of admissibility with a mathematically good definition, even if the traded assets are locally bounded. This is clearly shown by the Example 5.1 of Schachermayer. On the other hand, the new admissible class allows for a complete resolution of some important examples like the Merton problem in Section 4, for which no economically meaningful definition of admissibility was available.

\section{Appendix: an Ansel-Stricker-type lemma and consequences}

Lemma 6.1. (similar to Strasser [Stra])

Let $\left(X_{t}\right)_{0 \leq t \leq T}$ be a sigma martingale. The following are equivalent

1. $\left(X_{t}\right)_{0 \leq t \leq T}$ is a supermartingale (true, not local)

2. $\left(X_{\tau}^{-}\right)_{0 \leq \tau \leq T}$ is $u . i$.

If either of these conditions holds, then $\left(X_{t}\right)_{0 \leq t \leq T}$ is also a local martingale

Proof. One direction is obvious, since the negative part of a supermartingale is a submartingale. In order to prove the other implications, we define $T_{n}=\inf \left\{t|| X_{t} \mid>n\right\}$ and consider the stopped process $X^{T_{n}}$.

We observe that the process $X^{T_{n}}$ is bounded from below by the integrable random variable $\Theta_{n}=$ $-n-\left(X_{T_{n}}\right)^{-}$(the integrability follows from the of uniform integrability of the negative parts of $X$ along stopping times). By a version of the Ansel-Stricker Lemma (attached below as Lemma 6.2 for completeness), this means that $X^{T_{n}}$ is a local martingale. It is well known that a local local martingale is a local martingale, so $X$ is, indeed, a local martingale. Now, since the negative part of $X$ is of class $(D)$, it is an easy exercise involving Fatou's lemma for conditional expectations to show that $X$ is actually a supermartingale.

Lemma 6.2. (Variant of the Ansel-Stricker Lemma) If $X$ is a sigma-martingale and $X_{t} \geq-W, 0 \leq$ $t \leq T$ where $W>0$ is a random variable such that $\mathbb{E}[W]<\infty$ then

1. $\left(X_{t}\right)_{0 \leq t \leq T}$ is a local martingale

2. $\left(X_{t}\right)_{0 \leq t \leq T}$ is a super martingale 


\section{References}

[AS94] J.P. Ansel and C. Stricker. Couverture des Actifs contingents et prix maximum. Annales Institute Henri Poincaré 30, 303-315, 1994.

[Bia04] S. Biagini. A new class of strategies and application to utility maximization for unbounded processes. Technical report of the University of Perugia (available at http://diec.ec.unipg.it/publish/biagini.phtml), 2004.

[BF05] S. Biagini and M. Frittelli, Utility Maximization in Incomplete Markets for Unbounded Processes. Finance and Stochastics 9, 493-517, 2005.

[BF07] S. Biagini and M. Frittelli. The Supermartingale Property of the Optimal Wealth Process for General Semimartingales. Finance and Stochastics, 11/2, 253-266, 2007.

[Bia08] S. Biagini, An Orlicz Spaces Duality for Utility Maximization in Incomplete Markets. Proceedings of Ascona 2005, Progress in Probability, Birkhauser 2008.

[BF08] S. Biagini and M. Frittelli, A Unified Framework for Utility Maximization Problems: an Orlicz Space Approach. Ann. Appl. Prob. 18/3, 929-966, 2008.

[CSW01] J. Cvitanić, W. Schachermayer, and H. Wang. Utility Maximization in Incomplete Markets with Random Endowment. Finance and Stochastics 5, 237-259, 2001.

[DS98] Delbaen F. and W. Schachermayer. The Fundamental Theorem of Asset Pricing for Unbounded Stochastic Processes, Math. Ann., 312, 215-250, 1998.

[6AU] Delbaen F., P. Grandits, T. Rheinlander, D. Samperi, M. Schweizer and C. Stricker. Exponential hedging and entropic penalties, Math. Finance, 12/2, 99-123, 2002.

[KS02] Y. Kabanov and C. Stricker. On the Optimal Portfolio for the Exponential Utility Maximization: Remarks to the Six-Authors Paper, Mathematical Finance 12/2, 125-134, 2002.

[Kal02] J. Kallsen. Derivative pricing based on local utility maximization, Finance and Stochastics, 6, 115-140, 2002.

[KS99] Kramkov D. and W. Schachermayer. A condition on the asymptotic elasticity of utility functions and optimal investment in incomplete markets, Ann. Appl. Prob, 9/3, 904-950, 1999.

[KS06] D. Kramkov and M. Sîrbu. On the two-times differentiability of the value functions in the problem of optimal investment in incomplete markets, Annals of Applied Probability, 16/3, 1352-1384, 2006.

[OZ07] M. Owen and G. Zitkovic, Optimal Investment with an Unbounded Random Endowment and Utility Based Pricing Methods. Mathematical Finance, forthcoming.

[Sato] K. I. Sato Lévy processes and infinitely divisible distributions. Cambridge studies in advanced mathematics, 68, Cambridge University Press, 2005.

[Sch01] W. Schachermayer. Optimal investment in incomplete markets when wealth may become negative. The Annals of Applied Probability 11/3, 694-734, 2001.

[Sch03] W. Schachermayer. A super-martingale property of the optimal portfolio process. Finance and Stochastics 4, 433-457, 2003.

[Sh95] A. N. Shiryaev. Probability. Springer Graduate Texts in Mathematics, 1995.

[Stra] E. Strasser. Necessary and sufficient conditions for the supermatingale property of a stochastic integral with respect to a local martingale, Séminarie de probabilités XXXVII: 16, 385-393, LNM 1832 (2003) . 